\title{
GT2012-68509
}

\section{CHARACTERIZATION OF AERODYNAMIC PERFORMANCE OF BOUNDARY-LAYER-INGESTING INLET UNDER CROSSWIND}

\author{
Meng-Sing Liou \\ Aeropropulsion Division \\ NASA Glenn Research Center \\ Cleveland, $\mathrm{OH} 44135$ \\ Email: meng-sing.liou@nasa.gov
}

\author{
Byung Joon Lee \\ NASA Postdoctoral Program \\ NASA Glenn Research Center \\ Cleveland, $\mathrm{OH} 44135$ \\ Email: mdo.bjlee@gmail.com
}

\section{ABSTRACT}

NASA has been studying future transport concepts, envisioned to be technically realizable in the timeframe of 20202030 , to meet environmental and performance goals. One concept receiving considerable interest involves a propulsion system embedded into a hybrid wingbody aircraft. While offering significant advantages in fuel savings and noise reduction by this concept, there are several technical challenges that are not encountered in the current fleet and must be overcome so as to deliver target performance and operability. One of these challenges is associated with an inlet system that ingests a significantly thick boundary layer, developing along the wingbody surface, into a serpentine diffuser before the flow meeting fan blades. The flow is subject to considerable total pressure loss and distorted at the fan face, much more significantly than in the inlet system of conventional aircraft. In our previous studies [1],2], we have shown that through innovative design changes on the airframe surface, it is possible to simultaneously increase total pressure recovery and decrease distortion in the flow, without resorting to conventional penalty-ridden flow control concepts, such as vortex generator or boundary layer bleeding/suction. In the current study, we are interested in understanding the following issues: how the embedded propulsion system performs under a crosswind condition by studying in detail the flow characteristics of two inlets, the baseline and another optimized previously under the cruise condition. With the insight, it is hoped that it can help in the follow-on study by devising effective strategies to minimize flow distortion arising from the integration of an embedded-engine system into an airframe to the level acceptable to the operation of engine fan.

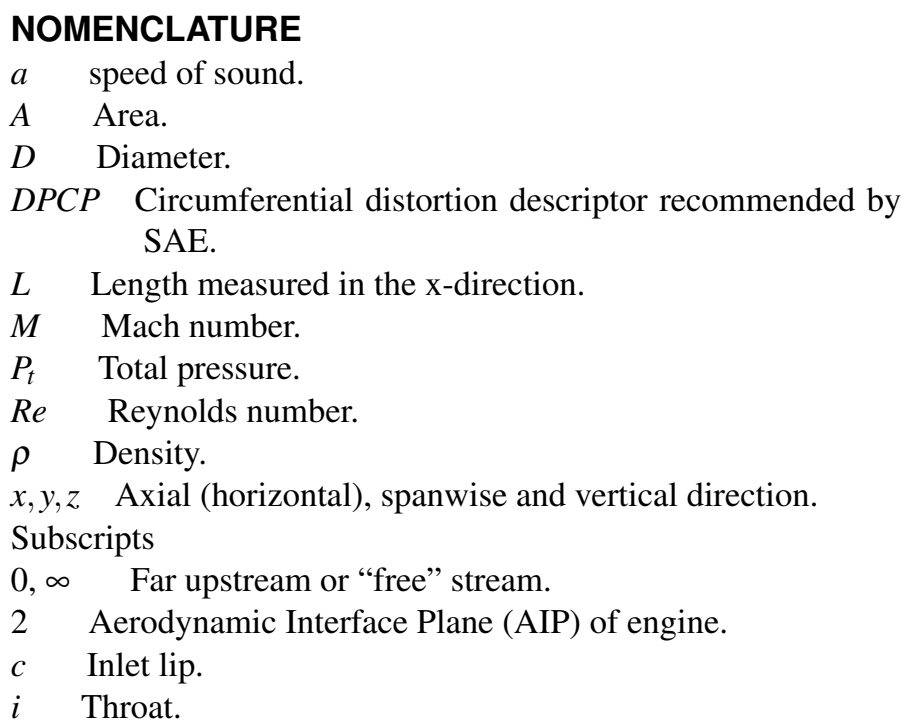

\section{INTRODUCTION}

NASA, under the current Fundamental Aeronautics Program (FAP), is considering several commercial transport configurations beyond current aircraft in the Subsonic Fixed Wing (SFW) Project [3], with objectives to achieve considerable improvement in performance and environmental impacts. Table 1 summarizes the specific goal metrics targeted to reduce noise, NOx emission,

Copyright (C) This material is declared a work of the U.S. Government and is not subject to copyright protection in the United States.

Approved for public release; distribution is unlimited. 
and fuel consumption before 2030. To achieve these demanding goals, non-conventional concepts are called for; but technology gap is too big that it requires evolutionary approach by focusing various concepts and technologies needed in the next three generations of aircraft, respectively named as $\mathrm{N}+1, \mathrm{~N}+2$, and $\mathrm{N}+3$. Noticeably, considerable reduction in each category of 1 is required in $\mathrm{N}+2$ (relative to Boeing 777-200 and GE90 engines) and $\mathrm{N}+3$ (relative to Boeing 737-800 and CFM56-7B engines). In this study, concepts for $\mathrm{N}+2$ is our interest. A concept that has potential to achieve these metrics and has been under intensive study is the hybrid wing body (HWB) airframe with a tightly integrated propulsion system, see Fig. 11 The inlet is non-circular at the entrance and the entering flow, no longer uniform or free of disturbances, and is now carrying with it a boundary layer developing along the fuselage; the inlet is thus known as boundarylayer-ingesting (BLI) inlet.

TABLE 1: NASA'S SUBSONIC FIXED WING PROJECT.

\begin{tabular}{|c|c|c|c|}
\hline \multirow{2}{*}{$\begin{array}{l}\text { TECHNOLOGY } \\
\text { BENEFITS* }\end{array}$} & \multicolumn{3}{|c|}{$\begin{array}{c}\text { TECHNOLOGY GENERATIONS } \\
\text { (Technology Readiness Level = 4-6) }\end{array}$} \\
\hline & $\mathrm{N}+1$ (2015) & $\mathrm{N}+2\left(2020^{\star *}\right)$ & $\mathrm{N}+3(2025)$ \\
\hline $\begin{array}{c}\text { Noise } \\
\text { (cum margin rel. to Stage 4) }\end{array}$ & $-32 \mathrm{~dB}$ & $-42 \mathrm{~dB}$ & $-71 \mathrm{~dB}$ \\
\hline $\begin{array}{l}\text { LTO NOx Emissions } \\
\text { (rel. to CAEP 6) }\end{array}$ & $-60 \%$ & $-75 \%$ & $-80 \%$ \\
\hline $\begin{array}{l}\text { Cruise NOx Emissions } \\
\text { (rel. to } 2005 \text { best in class) }\end{array}$ & $-55 \%$ & $-70 \%$ & $-80 \%$ \\
\hline $\begin{array}{l}\text { Aircraft Fuel/Energy Consumption }{ }^{\ddagger} \\
\text { (rel. to } 2005 \text { best in class) }\end{array}$ & $-33 \%$ & $-50 \%$ & $-60 \%$ \\
\hline
\end{tabular}

The concept of ingesting boundary layer into a propulsion system is not entirely new, originated in 1947 by Smith and Robert [4], who concluded the performance advantages with a significant longer range by submerging turbojet engines into the wing or fuselage. Subsequently further analyses were carried out at the Douglas Aircraft Company to assess the benefits of boundary layer ingestion to propulsion efficiency [5,6]. In 2002, Liebeck [7] chronicled the development of the blended wing body, in which the boundary-layer-ingestion propulsion system, assuming it was possible to provide a uniform flow and efficient pressure recovery at the fan face. Because of recent demands in reducing noise and fuel consumptions, renewed interest in embedded propulsion systems has arisen, significant efforts and investigations have been invested by various institutions, such as NASA's SFW project, Cambridge-MIT's Silent Aircraft Initiative [8], and European Commission's New Aircraft Concepts Research (NACRE) [9] and many reports have been produced, e.g., [10, 11, 12, 13, 14], documenting benefits and technology barriers and risks before the concept is realized in future com- mercial transport. Especially, rich depository of studies reports can be found from the NASA and SAI, in which various specifications are put forth and configurations published.

To fully reap the advantages of a BLI propulsion system, it must be placed close to the airframe so that no discernible wakes arise individually from them. Thus, it requires a geometrical change from being non-circular transitioning to circular and offsetting of its centerline, as shown in Fig. 1 and 2, which is designated as N2B concept. As depicted in Fig. 11 the configuration is designed to have three separate engine systems, with the center one situated further downstream from the side ones. Each nacelle will house a turbojet engine in the center flowpath, while the two side flowpaths will only include respectively a fan driven by the core engine, as suggested in Fig. 2.

As a result of mutual interference between flows over the airframe and nacelle and entering the inlet, the flowfield becomes enormously complex; asides from being turbulent, it typically involves flow separation, 3D vortical flow and possibly unsteadiness, in the inlet duct. This results in an increased nonuniformity, total pressure loss and distortion, which lead to additional concerns about blade mechanical loads, fan stall and even engine surge, and engine noise. Moreover, because the design of the BLI system is targeted at cruise conditions, new aerodynamic characteristics at off-design conditions arise and may have more significant impacts than the conventional aircraft configuration. A particular concern is about the aerodynamic performance in the BLI inlet when under the influence of crosswind at low speed conditions during takeoff and landing. This is the subject of the present study.

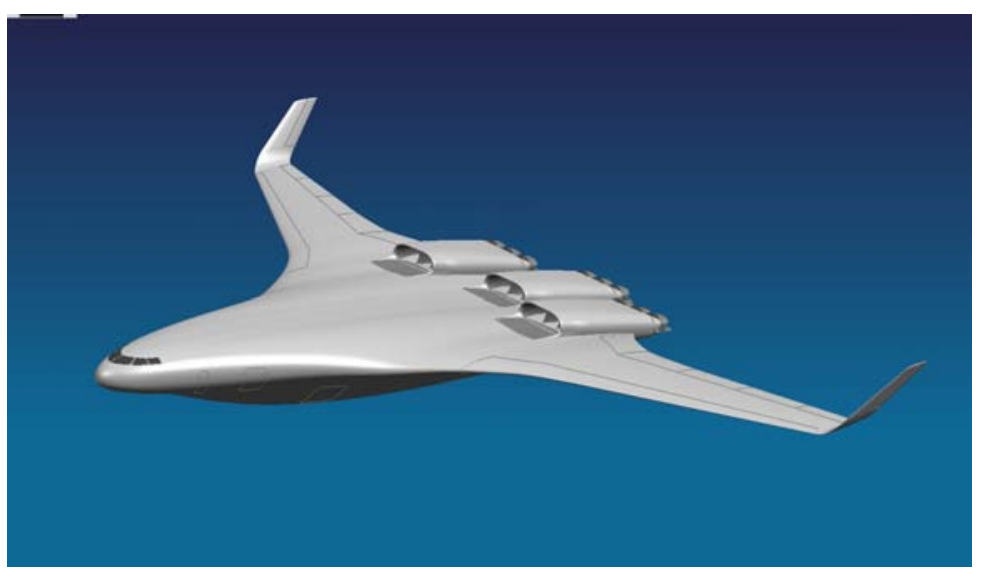

FIGURE 1: N2B CONCEPT CONFIGURATION [3].

Past studies of flow in BLI inlet include experimental and computational works, such as [15] and [16, 17] for inline and offset (S-shape) inlets, in which various flow control concepts were explored so as to reduce flow distortion. With consider- 


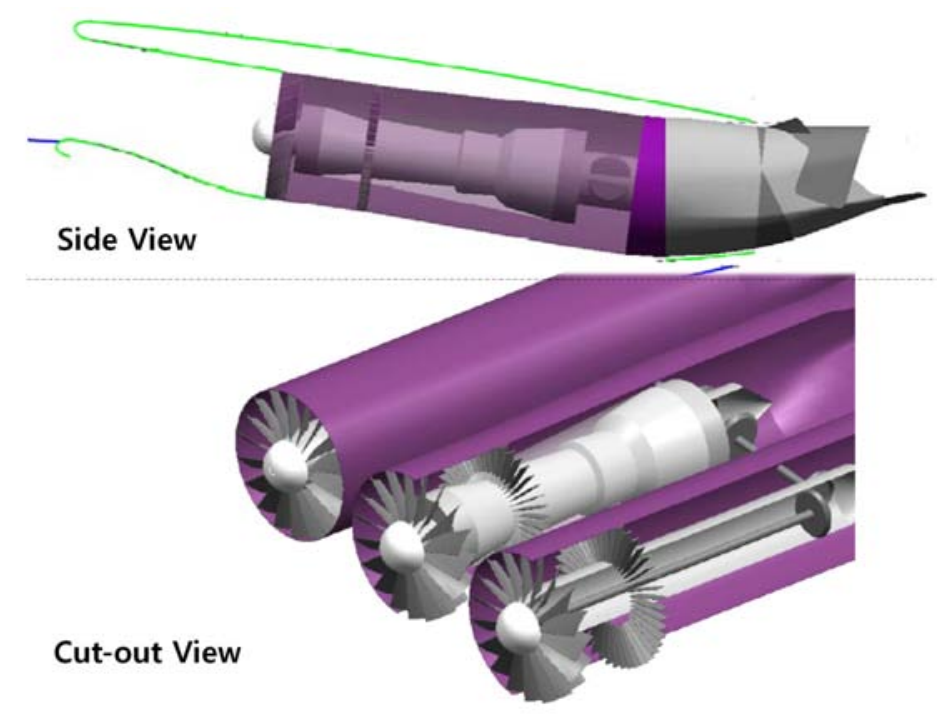

FIGURE 2: PROPULSION SYSTEMS AND NACELLE CONFIGURATION IN N2B.

able amount of ingested boundary layer (typically $30 \%$ of inlet height), distortion at aerodynamic interface plane (AIP) inevitably exceeds the level tolerable by the engine fan, thereby necessitating controlling, even reducing of boundary layer growth. Various flow control concepts have been studied for inlet applications; most chose vortex generators (VGs) [18, 15, 19], and some used active control [20,11] or bleeding. However, they all incur losses and costs, such as depriving of useful air supply, requiring additional power, keeping intact of VGs and bleed holes, etc. Moreover, determining the location, number, size and shape of VGs to be employed for achieving optimal results and maintaining the same level of effectiveness for a wide range of operating conditions has proven to be difficult because the number of design variables is large and the flow is extremely complicated. A systematic approach to quantitatively defining the above factors is perhaps the only robust route. The design of experiment method has been used in [18, 19]. Recently, genetic algorithms (GAs) together with data mining were employed to determine the locations and orientations of vortex generators so as to effectively reduce distortion and increase total pressure recovery [21]. While GAs have several advantageous features, including ease of implementation and allowing searching for a global optimal point, they are computationally expensive for a large problem. In another study [2], we chose the adjoint sensitivity approach for minimizing distortion by redesigning the airframe surface to "condition" the approaching boundary layer to the inlet. With this new intriguing surface shape, not only is the distortion at the AIP significantly reduced, but also the total pressure recovery increased considerably. These benefits are realized only through a fundamental change in flow structures, without incurring addi- tional mechanical devices or loss of useable compressed air for boundary layer bleeding.

With the operability of using the BLI concept demonstrated for the design condition, we now shall focus on understanding the flow characteristics at off-design conditions, of special importance is to assess the performance under crosswind at low speed flight during takeoff and landing. This subject has received much less attention than that for the cruise condition, rightly so because an aircraft spends its vast majority of time in cruising. However it is hugely important to ensure safety during this very brief, yet critical, phase of the flight. Safe landing and takeoff may be required with crosswind speed on the order of 15-20 m/s (roughy 30-40 knots) and the associated sideslip angle may be of 15 to 20 degrees for conventional transport. Recently, several detailed experimental and computational analyses have been reported, see e.g. [22, 23, 24]; they all focus on an isolated nacelle at "zero" cruise speed and subject to crosswind. Severe flow separations inside and outside the nacelle, resulting in significant distortion to the flow before AIP, are observed. Considerable hysteresis is encountered as the crosswind changes angles or magnitude [23]. The deterioration in performance is expected to be even worse in the case of BLI inlet. It is unclear how the performance deteriorates with respect to relevant factors, such as crosswind angle, magnitude, flight speed, etc. Thus, it is an even more important step to make a definitive quantitative analysis and to devise feasible practical changes to the embedded nacelles/inlet and the airframe surface surrounding it. In this paper, we shall take the first step to characterize the resulting flow phenomena and assess its aerodynamic implications at the AIP so that these findings will lay the foundation and provide the direction for the subsequent optimal redesign.

The remainder of the present paper will cover the following topics: (1) Choice of CFD requirements for accurately evaluating the complex flowfield in the BLI system, (2) Statement of the problem considered in this study (3) Validation of the CFD capability for capturing key aerodynamic performance metric, (4) Aerodynamic performances of the original and optimal designs at the cruise condition, (5) Characterization of aerodynamics under off-design crosswind condition, and (5) Future research.

\section{CHOICE OF CFD REQUIREMENTS}

The N2B configuration is designed to fly at high subsonic speed and the boundary layer ingested into the inlet is turbulent. The CFD capability needed for reliably handling this type of flow should possess two main technical elements: (1) reliability for accurately predicting complex turbulent flow from low to cruise speeds, and (2) flexibility for resolving different geometry topologies and viscous layers without sacrificing grid quality (orthogonality and mesh spacing). The analysis is based on solving three-dimensional Reynolds-averaged Navier-Stokes equation with closure by the two-equation $\kappa-\omega$-SST model [25]. 
The basic CFD code has been validated for many problems in the past, as documented in the $\mathrm{PhD}$ dissertation by Lee [26]; it has been further augmented and comparison with experimental data for the boundary layer ingestion inlet has been verified in our previous study [2]. For the present study with emphasis on crosswind condition, low Mach flow dominates in regions internal and external to the nacelle, for which the conventional CFD schemes are afflicted with significant numerical dissipation, thereby contributing to inaccuracy and deterioration in convergence. An advanced upwind method, $\mathrm{AUSM}^{+}$-up [27], capable of handling flow from low to high speed regimes, has been implemented in the code. The time discretization is accomplished by the implicit LU-SGS scheme [28]. Since the $\kappa-\omega$-SST model is regarded as the state of the art model for a wide range of flow phenomena, no attempt in this study to consider other models or other formulations such as large-eddy simulation, as this subject matter is beyond the scope of the current investigation. It is noted that the variables presented are non-dimensionalized, unless indicated otherwise; the density, velocity and pressure are nondimensionalized by the freestream quantities, $\rho_{\infty}, a_{\infty}$, and $\rho_{\infty} a_{\infty}$.

The need for flexibly handing complex geometry can be realized by utilizing the overset grid strategy [29], in which two or more grid blocks overlap and each has an independently generated grid, thereby allowing a better control of grid quality and flexibility for adapting to geometry.

\section{PROBLEM STATEMENT: CFD SOLUTION OF BLI INLET FLOW}

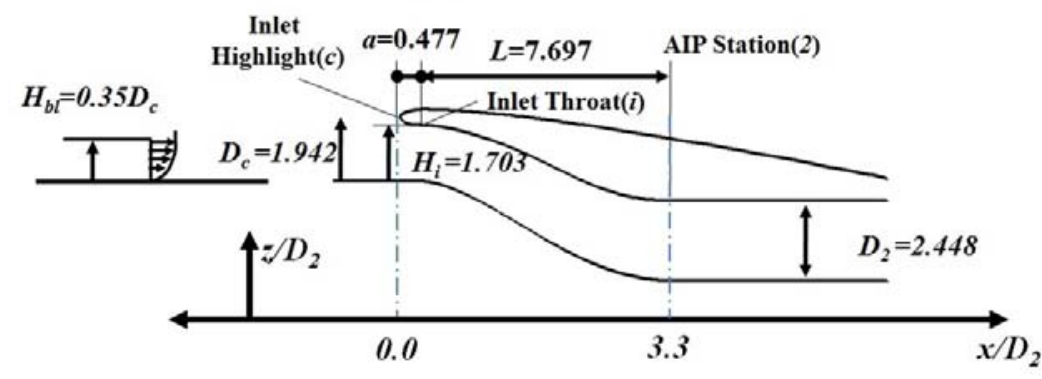

FIGURE 3: SCHEMATIC OF THE BASELINE BLI INLET UNDER STUDY: A SIDE VIEW SHOWING INCOMING BOUNDARY LAYER AND PRIMARY GEOMETRICAL NOTATION. ALL DIMENSIONS ARE IN INCHES, DRAWINGS NOT TO SCALE, X-COORDINATE IS ALIGNED WITH THE SYMMETRY PLANE AND $\mathrm{X}=0$ COINCIDES WITH THE INLET HIGHLIGHT. [17]

In this study, we consider a circular type BLI inlet (desig- nated as type A in [17] 1 ; this inlet has been extensively studied experimentally and numerically. Its geometry is schematically shown in two views in Fig. 3, together with relevant dimensions, where stations $c, i$, and 2 respectively referring to the lip (highlight), throat, and AIP of the inlet. The X-Z plane coincides with the inlet symmetry plane with the $\mathrm{X}$-coordinate pointing downstream. the inlet highlight is defined at $X=0$. The key geometric parameters are: the diameter at AIP $D_{2}=2.448$ in, the throat is located at $X_{i} / D_{2}=0.1949$ and the AIP at $X_{2} / D_{2}=3.339$, the throat height $H_{i} / D_{2}=0.6957$, and the area ratio $A_{2} / A_{i}=1.070$. The offset of the inlet is $0.7735 D_{2}$. The figure also illustrates schematically an incoming boundary layer to the inlet, with an approximate thickness of $30 \%$ of entrance height. We note that in most line plots we interchangeably use $D$ for $D_{2}$.

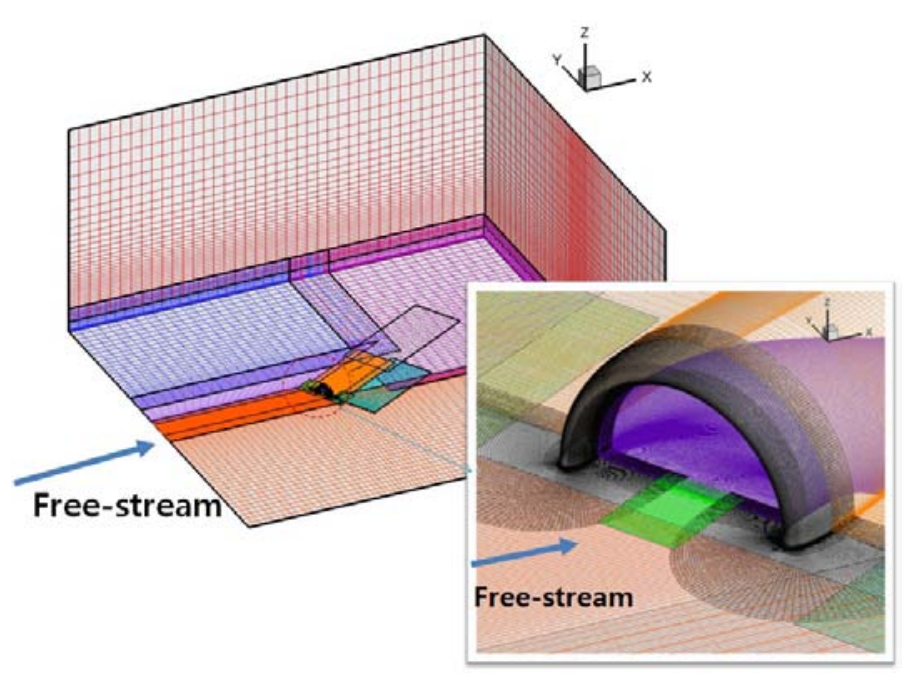

FIGURE 4: COMPUTATION DOMAIN USED IN THIS STUDY AND AN ENLARGED VIEW OF THE INLET ENTRANCE, SHOWING THE OVERSET GRID SYSTEM, EACH COLOR DENOTING AN INDIVIDUAL BLOCK GRID.

To encompass flow domains inside and outside the inlet and allow accurate resolution of viscous layers, using overset grids has advantages in generating quality grid and dealing with different topologies with ease. The former becomes especially attractive in shape optimization since the grid will be changing throughout the optimization process. Figure 4 shows the computation domain used in this study and an enlarged view near the BLI inlet entrance, depicting the overset mesh system, con-

\footnotetext{
${ }^{1}$ While the inlet entrance in N2B is more of super-elliptic shape, it is believed that qualitative phenomena in both cases are similar and more importantly the $\mathrm{N} 2 \mathrm{~B}$ inlet is still a subject of conceptual study and there is no data available yet for validation.
} 
taining 16 grid blocks, 7 configuration blocks and 9 background blocks, in which individual grid blocks are designed to resolve the curvature of inlet lip, the "inviscid" region outside the inlet, the core region inside the inlet background, and the boundary layer near the inlet entrance, the viscous region next to the inlet wall. The entire mesh has about 10.7 millions grid points in total and more than doubles the grid used in the previous study where only a half domain is needed by employing symmetry since no crosswind is involved.

As the main interest is to investigate how well the BLI inlet performs under the crosswind condition. The condition considered critical is during takeoff and landing phase. The crosswind strength is specified to be 40 knots $(20.78 \mathrm{~m} / \mathrm{s})$ and the sideslip angle is 30 degrees. At the sea level dry air condition, this yields a crosswind Mach number of 0.06 and freestream Mach number of 0.1212 . The boundary-layer thickness at the entrance of the inlet is targeted to be $0.35 H_{c}$, an expected value in a realistic flight situation. 2 Short of knowing the boundary-layer flow on actual configuration, we assumed that a turbulent flow is developing on a flat plate at a distance of $20 D_{2}$ upstream of the inlet, such that the required boundary-layer thickness is obtained. Since the flow at AIP is subsonic, as required for the fan operation, the usual strategy of imposing static pressure there is used. However, mass flow rate is the parameter customarily known in engine operation, instead of static pressure. This can be bridged by assuming a locally 1D isentropic condition, yielding a direct relationship between mass flow rate and static pressure (e.g., see [1]).

The outflow condition is specified at a somewhat arbitrary interface plane with the fan, if the interaction between inlet and fan is neglected in the analysis. The computational condition at this "virtual" AIP can be handled several ways, such as specifying a mass flow rate or static pressure. Since there is no known information about how the static pressure distribution would be radially, it is important to minimize influence of any a priori specification of its value and profile. Hence, the computation domain is extended beyond AIP with additional straight section of same diameter and of length $L_{i 2}=\left(X_{2}-X_{i}\right)$. It is verified after the solution is converged that in this extended section the flow becomes nearly unchanged towards the end and the static pressure becomes uniform, but the pressure at AIP still has radial as well as circumferential variations.

\section{VALIDATION AND COMPARISON WITH DATA AT DE- SIGN CONDITION}

To ensure the predictive capability for dealing with complexities relevant to the problem of our interest, we have carried out extensive validation tests and the results were documented previ-

\footnotetext{
${ }^{2}$ It is assumed that there is no spanwise variation in the flow approaching the inlet since the inlet is attached to an infinite plate upstream. In actual aircraft, the wingbody will have a spanwise variation in shape, thus adding another dimension of complexity to the flow.
}

TABLE 2: COMPARISON OF COMPUTED INLET PERFORMANCE WITH MEASURED DATA.

\begin{tabular}{lccc}
\hline Reynolds number & $\begin{array}{c}\text { Total pressure } \\
\text { recovery ratio }\end{array}$ & Distortion & $\begin{array}{c}\text { Mass flow ratio } \\
A_{o} / A_{c}\end{array}$ \\
\hline $3.8 \times 10^{6}[17]$ & 0.952 & 0.0540 & 0.534 \\
$3.8 \times 10^{6}$ present CFD & 0.956 & 0.0596 & 0.533 \\
$2.2 \times 10^{6}$ present CFD & 0.943 & 0.0630 & 0.527 \\
\hline
\end{tabular}

ously in [1]. From Table 2, it is evident that the employed CFD code is capable of providing a reliable solution insofar as the inlet performance metric is concerned. A lower Reynolds number condition was also computed because it was suggested in a previous study by Berrier and Allan [30] that this lower value gave a closer agreement in pressure distribution on the bottom wall. However, this table indicates that (1) lowering Reynolds number produces less total pressure recovery, higher distortion and lower mass flow ratio, this higher-loss phenomenon suggests an existence of a larger separated region, as manifested by the fact of a flatter pressure rise. However, with this choice of lower Reynolds number, the inlet metric deviates noticeably from the data. Hence we conclude that the discrepancy in surface pressure distribution might have to be attributed to other factors.

\section{OPTIMIZATION ON CRUISE CONDITION}

Shape optimization of the inlet bottom wall has been carried out in our previous studies [1,2] based on the adjoint sensitivity formulation to minimize flow distortion $D P C P_{a v g}$ at AIP on cruise condition: $M_{\infty}=0.85, R e_{\infty}=3.8 \times 10^{6} . \quad D P C P_{a v g}$ is a circumferential distortion descriptor, defined according to the SAE standard [31] by averaging total pressure distortions over 5 equally area-weighted rings:

$$
D P C P_{\text {avg }}=\frac{1}{5} \sum_{i=1}^{5}\left\{\frac{\left(\bar{P}_{t, i}-\bar{P}_{t, i_{\text {low }}}\right)}{\bar{P}_{t, i}}\right\}
$$

where $\bar{P}_{t, i}$ represents the average over the 12 equally spaced points on ring $i$ and $\bar{P}_{t, i_{l o w}}$ is the average of total pressures below $\bar{P}_{t, i}$.

Here we include representative and revealing results, which demonstrate a considerable simultaneous improvement in distortion reduction and total pressure recovery. The interested reader is encouraged to find details in the cited references.

Figure-5 displays the "oil-flow" patterns of both designs; the original design gives rise to a significant "push" of streamlines from sidewalls, because of low-momentum-fluid blockage, while the streamlines in the optimal design follow the contours of in- 
let. These are a result of making a series of "sinusoidal-like" of varying amplitudes and wave lengths modulations on the bottom wall, in a patch defined by

$$
-1.8 \leq \frac{X}{D_{2}} \leq 0.5 \text { and }-1.0 \leq \frac{Y}{D_{w}} \leq 1.0
$$

where $D_{w}(x)$ is the width of crosssection at location $x$

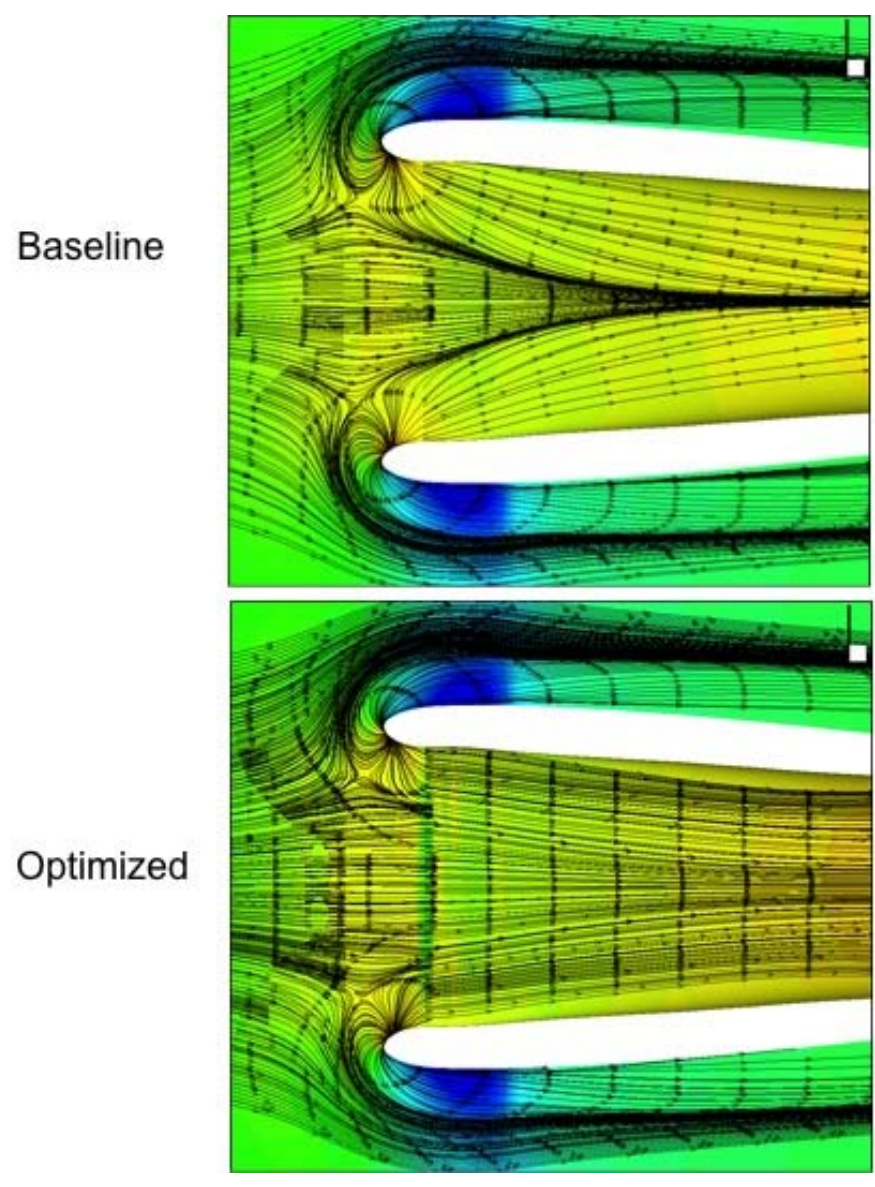

FIGURE 5: “OIL FLOW" PATTERN OF THE ORIGINAL (TOP) AND OPTIMAL (BOTTOM) INLETS, SUPERIMPOSED ON PRESSURE CONTOURS. [1,2]

Figure 6 shows that the optimized design has consistently in all off-design conditions far smaller distortion values, 50 to $70 \%$ less than the baseline values. Also notice that at the pressure ratio $P_{b} / P_{t, 0}=0.9353$ the baseline model approaches unstart with a much reduced mass flow ratio, but the optimized model appears still functioning. Furthermore, the total pressure contours at AIP shown for two mass flow ratios also confirm the superiority in pressure recovery after optimization.

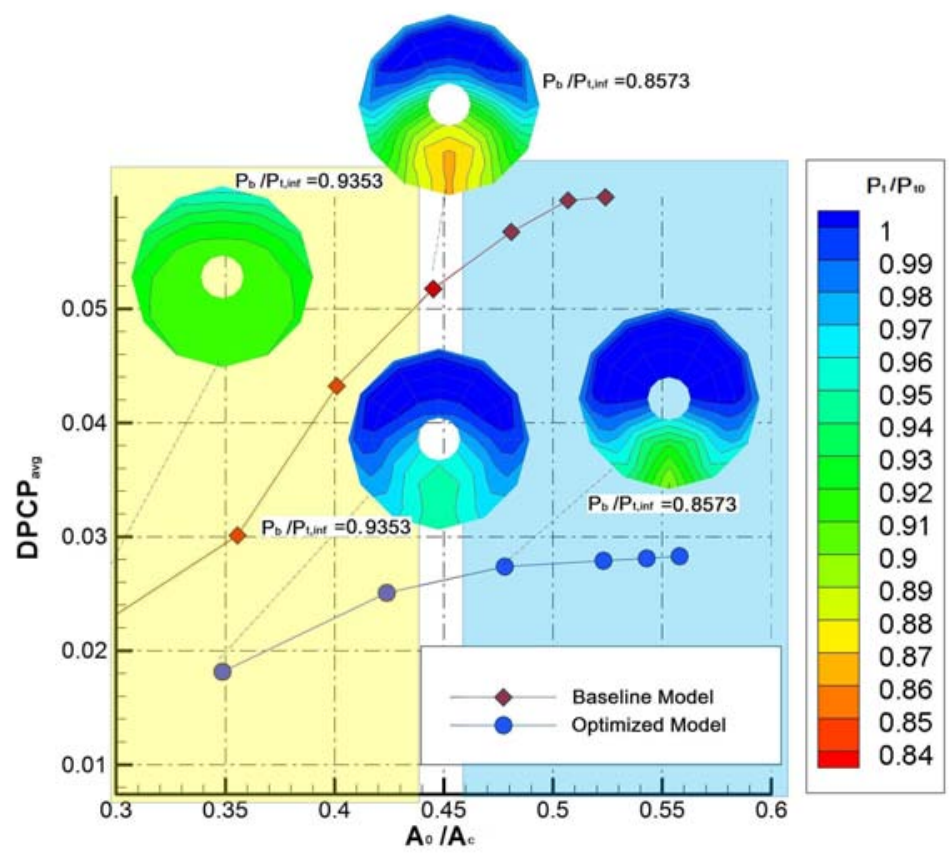

FIGURE 6: COMPARISON OF FLOW DISTORTIONS AT OFF-DESIGN CONDITIONS. [1,2]

In what follows, we present the resulting performance of the inlet in terms of $D P C P_{a v g}$, total pressure recovery $\left(P_{t} / P_{t, \infty}\right)$, and flow rate, but at takeoff low speed with crosswind.

\section{CROSSWIND CONDITION: CHARACTERIZATION OF AERODYNAMIC PERFORMANCE}

As seen above, redesigning the airframe surface near and ahead of the inlet entrance has achieved the goal of improving the inlet aerodynamic performance to the extent tolerable to the fan. It is now to ensure that the BLI inlet deliver needed performance under off-design conditions, especially with regard to safety under severe conditions, such as takeoff and landing with crosswind. As indicated earlier, the condition considered is: crosswind Mach number of 0.1 and sideslip angle of $30 \mathrm{de}-$ grees, resulting in $\operatorname{Re}_{\infty, D_{2}}=0.58 \times 10^{6}$. For this analysis, we can no longer use a half plane by assuming symmetry, the complete nacelle/inlet must be considered, see the overset grid system in Fig. 4 The back pressure ratio is set as $P_{b} / P_{\infty}=0.7373$. The approaching boundary layer is generated to give a thickness of $33 \%$ of highlight height at the inlet entrance. Fine grid spacings are ensured near a solid surface in each grid block so that viscousdominated regions are adequately resolved; a representative wall distance of a first grid point measured by $y^{+}$is on the order of unity, specifically at the inlet entrance it is less than one.

In what follows, we shall not only focus on the performance 
of the baseline BLI inlet and the underlying flow characteristics, but will also accompany with those from the inlet optimized for the cruise condition [1, 2], so that we shall give a quantitative assessment as to whether the previous optimization holds its advantages over the baseline geometry under the current crosswind condition. For the sake of conveniently referring to this geometry, we shall here afterwards use the term "optimized" inlet to mean the geometry previously optimized for the cruise condition [1, 2], but it should be emphasized that it is not optimized for the current crosswind condition.

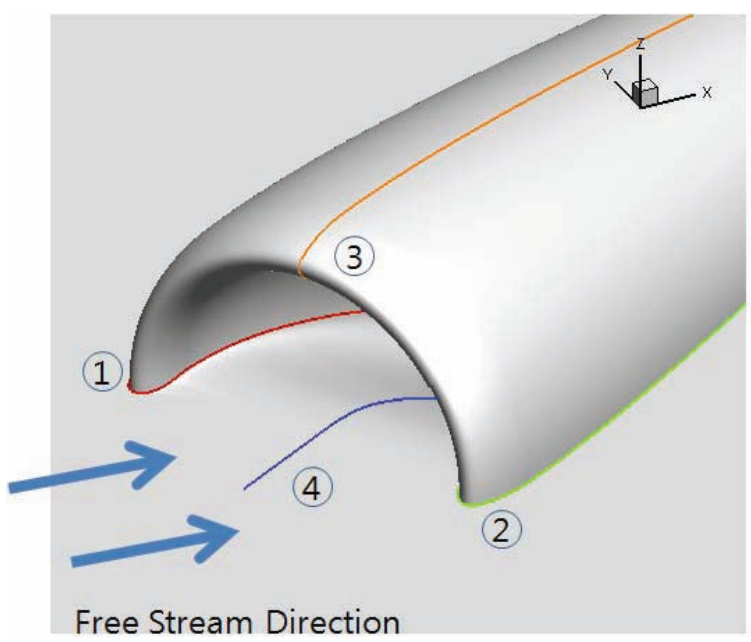

FIGURE 7: LOCATIONS OF "NUMERICAL" PRESSURE RAKES FOR SHOWING PRESSURE DISTRIBUTIONS IN THE FOLLOWING FIGURE.

First, we present the surface pressure distributions on four representative circumferential sections, as designated in Fig. 7 , on both internal and external surfaces of the inlet. The surface between lines 2 and 3 will be denoted as leeward side and the opposite (between lines 1 and 3 ) is the windward side; the surface 1-4-2 is the bottom surface of the inlet, which eventually evolves into the lower half of the circular duct. It is evident that there are considerable variations in the circumferential direction, between the internal and external surfaces. The pressure distributions on the internal and external surfaces on these four sections are displayed in Figs. 8 and 9 respectively. On the windward side (section 1) the external surface has a stagnation point downstream the nacelle highlight, the pressure there, see top figure in Fig. 8, is only slightly greater than $P_{\infty}$ because the dynamic pressure is small due to a low speed, and it reduces to a constant value of essentially $P_{\infty}$ since the outer surface has no changes in slope or curvature. While entering the internal inlet, the flow however undergoes a rapid expansion as it negotiates around the lip and continues to "diffuse" downstream, accompanying with
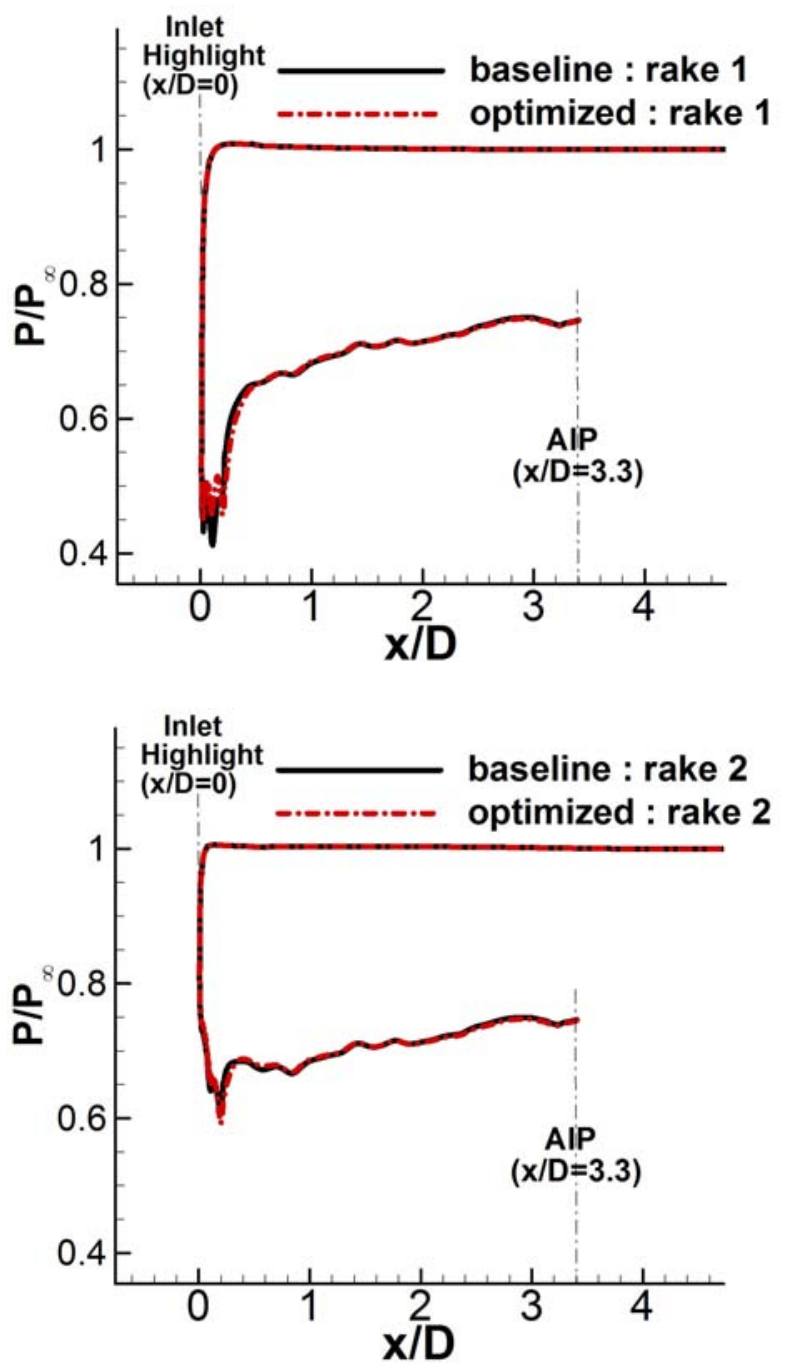

FIGURE 8: PRESSURE DISTRIBUTIONS ON CIRCUMFERENTIAL SECTIONS, ALONG LINES 1 AND 2 (OPPOSING WINDWARD AND LEEWARD INLET/AIRFRAME JUNCTURES), ON INTERNAL AND EXTERNAL (HAVING HIGHER PRESSURE VALUES) INLET SURFACES.

increasing pressure, which could lead to flow separation (as will become evident later in Fig. 13). The external surface on the leeward side (section 2) yields a similar behavior as in the windward side, see bottom figure in Fig. 8, but the internal surface sees a much reduced expansion in pressure and the pressure rise downstream is milder, thereby less likely giving rise to flow separation. Also included in both plots are the results from the optimized inlet. The trends of pressure distributions between the baseline and optimized inlets in these two sections are very close, except a somewhat reduced pressure expansion in the windward side of the optimized inlet. 
Figure 9 reveals that the pressure distributions on the internal surfaces of the top section (section 3 ) and bottom section (section 4) are more complex than those just discussed; on section 3 we see an extended pressure decrease following the initial rapid rise near the throat and a final rise to the specified AIP pressure, unlike those observed in sections 1 and 2. Again, the results of both baseline and optimized inlets are nearly indistinguishable, as expected since the modification in geometry is made on the bottom wall only. However, the geometry effect is now observed on the bottom wall pressure (section 4 ), the effect remains well into the downstream region of the inlet, up to $X / D_{2} \approx 0.5$, where
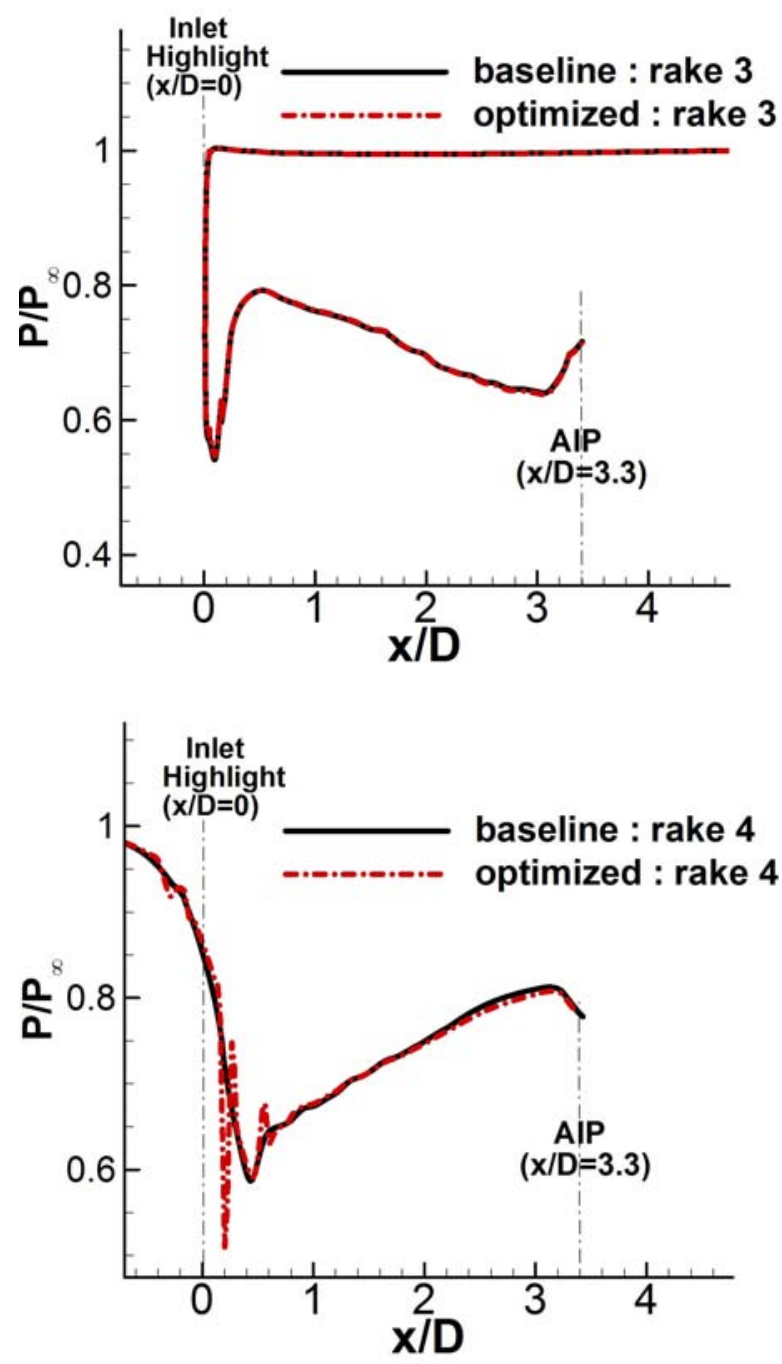

FIGURE 9: PRESSURE DISTRIBUTIONS ON CIRCUMFERENTIAL SECTIONS, ALONG LINES 3 AND 4 (OPPOSING TOP AND BOTTOM WALLS), ON INTERNAL AND EXTERNAL (HAVING HIGHER PRESSURE VALUES) INLET SURFACES.
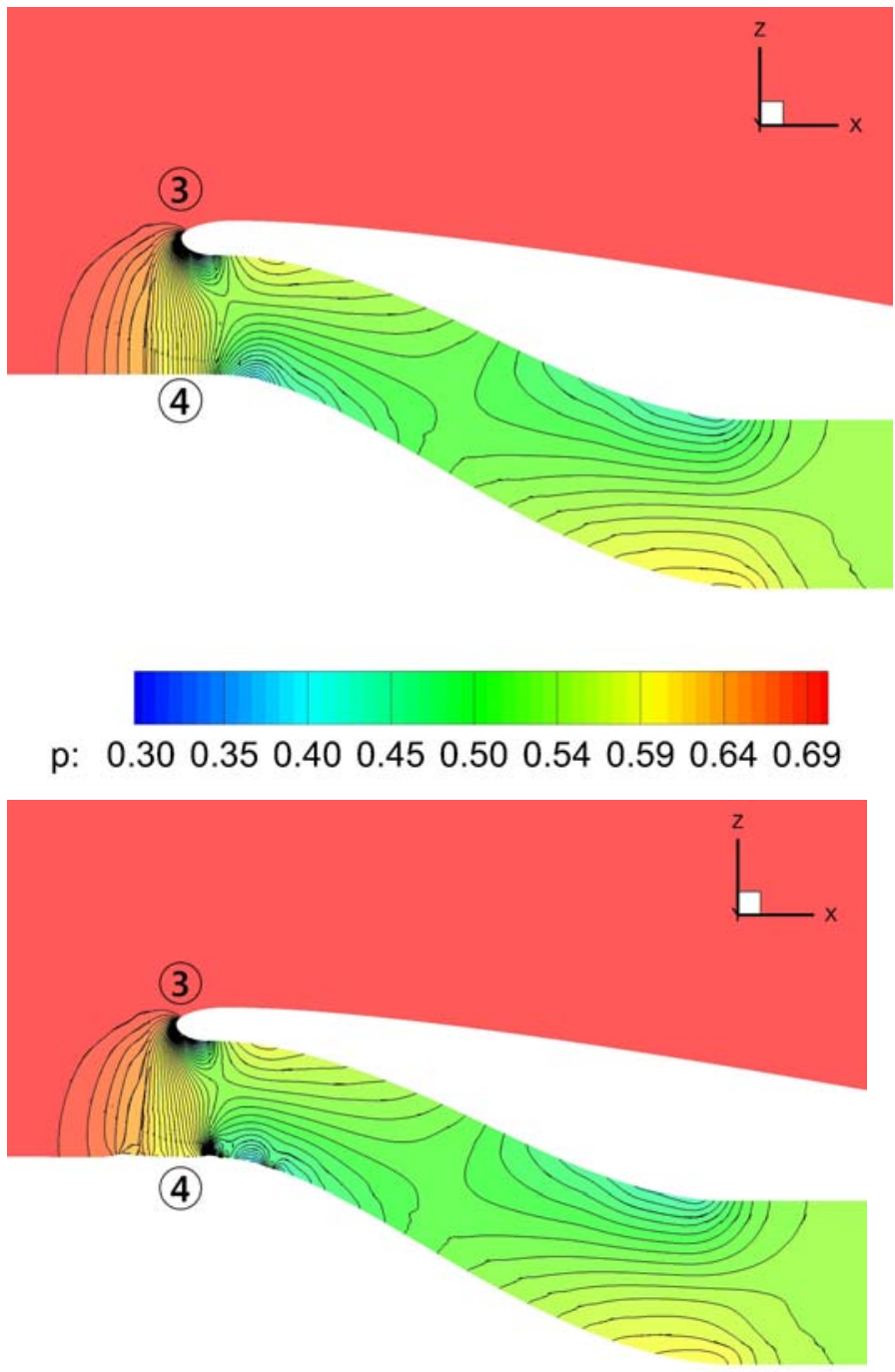

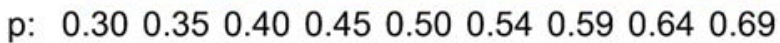

FIGURE 10: COMPARISON OF PRESSURE CONTOURS ON THE SYMMETRY PLANE INSIDE THE BASELINE AND OPTIMIZED INLETS.

the modification of wall shape terminates. The flow here behaves differently from that on section 3; the pressure has a milder expansion initially and then steadily climbs till the AIP.

Next in Figs. 10, we show the pressure contours on the symmetry plane; the flow is accelerating primarily inside the inlet for this flow configuration, as also revealed in previous figure Fig. 9 and the Mach number contours (not shown) remains by and large above 0.6 , only around the second bend on the bottom wall (near 
AIP) is its value lowered. The contours between the baseline and optimized inlets are almost the same, except on the bottom wall near the entrance where the wall is reshaped.
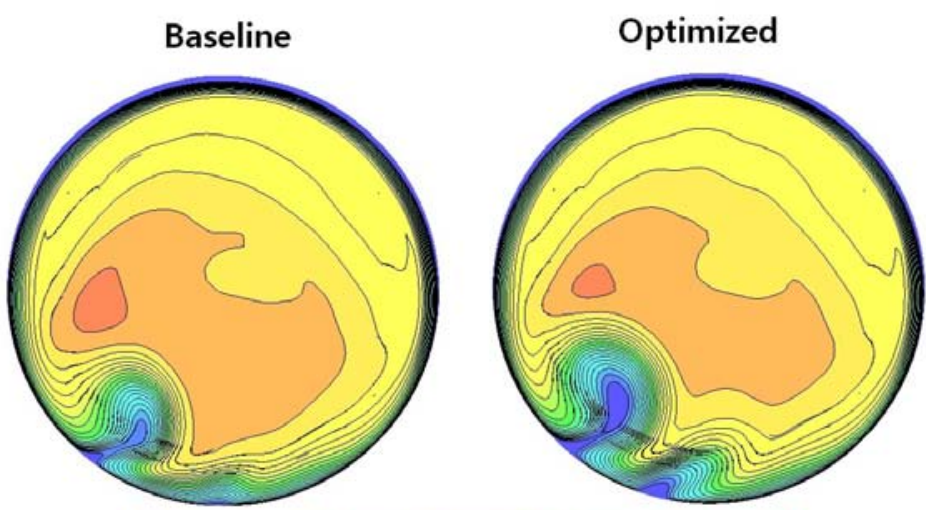

$\mathbf{P}_{\mathbf{t}} / \mathbf{P}_{\mathbf{t}, 0}:$

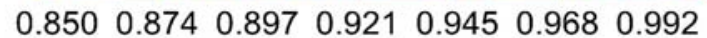

FIGURE 11: COMPARISON OF PRESSURE CONTOURS ATAIP OF THE BASELINE AND OPTIMIZED INLETS.

Figure 11 compares the total pressure contours at AIP of baseline and optimized inlets; both plots clearly exhibit a great deal of asymmetry, thereby contributing distortion, and have a low pressure region skewed towards the windward side of the nacelle. The pressure level in the optimized inlet however has lower value, suggesting a lower pressure recovery and higher distortion. The origin of this low pressure region can be traced to the flow entering the inlet, as shown in Fig. 12, where Mach number contours are displayed at several streamwise sections superimposed with particle traces inside the inlet. A pocket of low momentum fluid was observed in the optimized inlet, which in turn contributes to the thickening and diffusion of low momentum boundary layer fluid, thus losing total pressure along the way.

Table 3 summarized the performance metrics of both inlets, it shows that the optimized shape, as is, does not hold advantages under crosswind condition. However, this does not necessarily mean that optimization fails under this condition, it may require considering an extended design space, in our case it is the area upstream of inlet entrance in the spanwise direction. Nevertheless, the pressure recovery is only slightly deteriorated.

Examination of the velocity field at various cross-sections reveals interesting characteristics of the flow, the results of both
TABLE 3: COMPARISON OF PERFORMANCE BETWEEN BASELINE AND OPTIMIZED INLETS.

\begin{tabular}{lcc}
\hline Inlet & $\begin{array}{c}\text { Total pressure } \\
\text { recovery ratio }\end{array}$ & Distortion \\
\hline Baseline & 0.963 & 0.0169 \\
Optimized & 0.958 & 0.0515 \\
\hline
\end{tabular}

baseline and optimized inlet are represented in Fig. 13 The flow is clearly asymmetrical with respect to the symmetry plane, due to the crosswind component. The cross-sectional velocity components (projection of the velocity vector onto a $\mathrm{x}=$ constant plane, leaving only the $\mathrm{y}$ and $\mathrm{z}$ components) clearly portraits a flow from the windward to leeward sides in the exterior of the nacelle; it also exhibits that a separated flow region, denoted as the front separation, first appears only in the windward stagnation juncture, see the plot at $X / D_{2}=0.5$. Meanwhile a second separated region is beginning to form, visible at $X / D_{2}=2.5$ on the top of the nacelle, roughly 135 degrees from the front stagnation point, and is growing in size, as clearly displayed at $X / D_{2}=3.3$. This second separated region does not appear connected to the front separation in the sense that it is not morphed from the latter. Also its size eventually decrease to zero further downstream

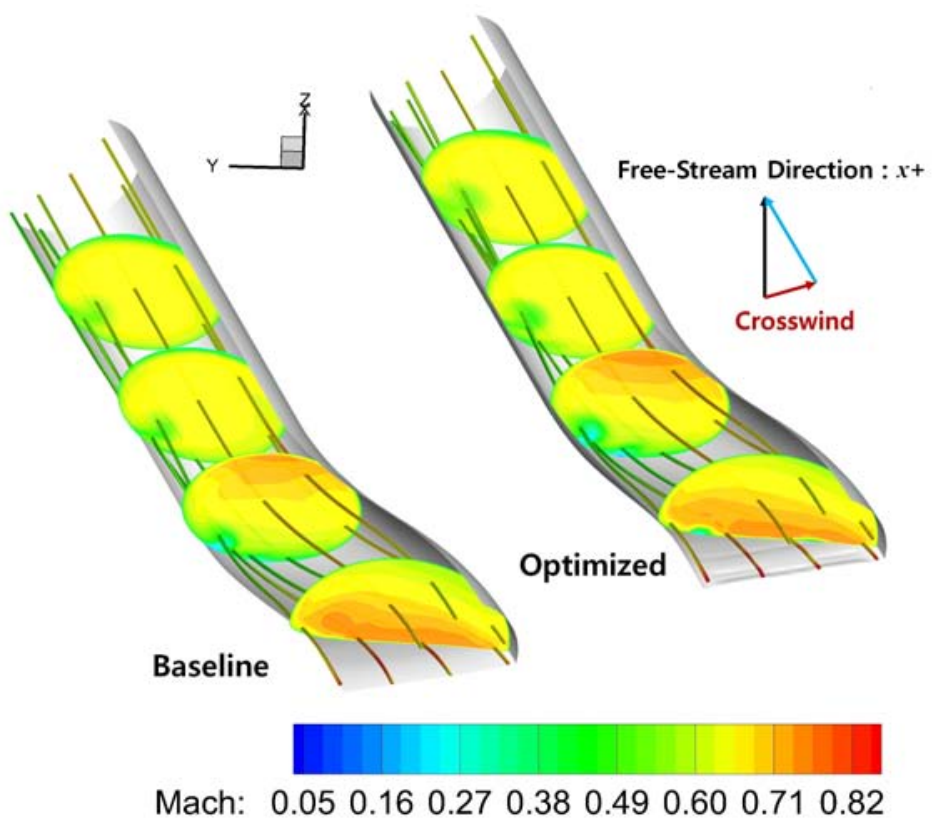

FIGURE 12: COMPARISON OF CROSS-SECTIONAL MACH NUMBER CONTOURS BETWEEN BASELINE AND OPTIMIZED INLETS. 


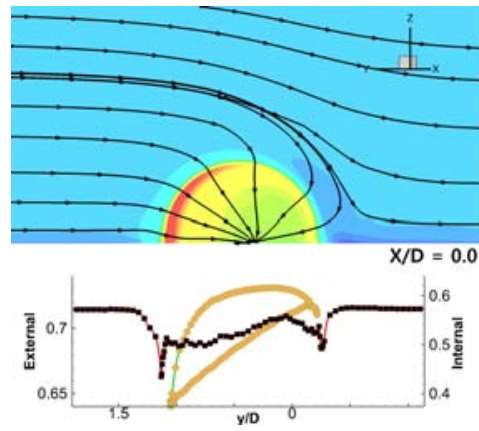

(a) $X / D_{2}=0.0$

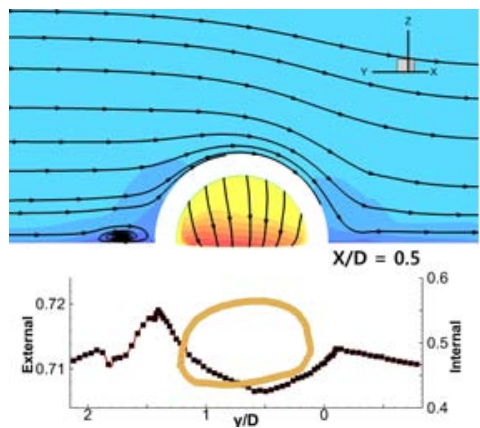

(c) $X / D_{2}=0.5$
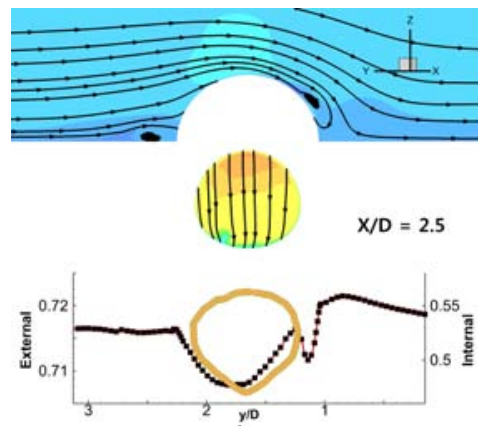

(e) $X / D_{2}=2.5$
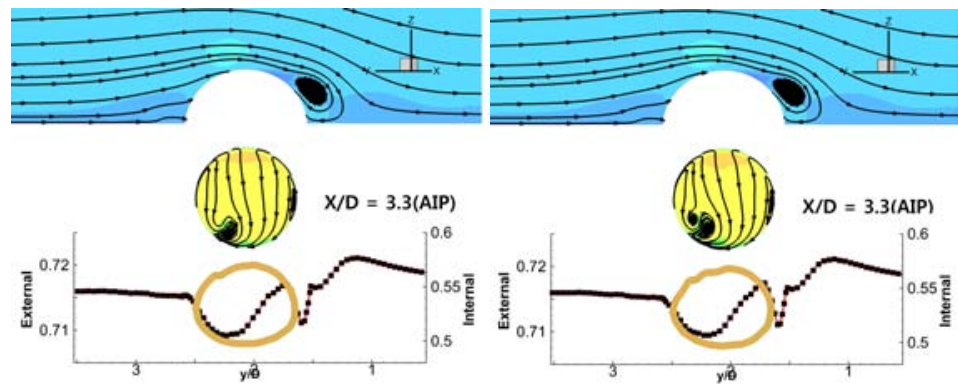

(g) $X / D_{2}=3.3$

C.1.

Mach: 0.020 .150 .290 .420 .560 .69

FIGURE 13: MACH NUMBER CONTOURS AT VARIOUS CROSS SECTIONS, WITH SUPERIMPOSED CROSS FLOW STREAMLINES, IN EXTERNAL AND INTERNAL FLOW DOMAINS OF THE INLET. LEFT COLUMN: BASELINE INLET; RIGHT COLUMN: OPTIMIZED INLET. at about $X / D_{2}=6.0$, because the nacelle eventually completely submerges into the airframe and the external flow sees no obstacle, thereby returning to a flat surface and smooth flow. The exterior flow fields of both inlet are indistinguishable.

On the other hand, the behavior inside the inlet is an entirely different matter. At the inlet entrance $\left(X / D_{2}=0\right)$, the flow is also quite asymmetrical; but once inside the inlet, the flow appears to symmetrize relatively quickly within a short distance, say roughly $X / D_{2} \leq 0.5$. However, a remnant of low-speed pocket from the entrance bottom wall first appears near the windward side of the inlet, first inkling seen at $X / D_{2}=0.5$ in the optimized inlet and clearly visible at $X / D_{2}=2.5$ in both inlets; this low speed flow is of larger size in the optimized inlet. The growth of this momentum region persists, displaying spanwise nonuniformity; a secondary flow develops as the geometry undergoes a change from a noncircular shape to circular. Eventually at $\operatorname{AIP}\left(X / D_{2}=3.3\right)$, the vortex becomes sufficiently strong that a vortex appears at the lower corner of the inlet, whereas a counter-rotating pair exist in the optimized inlet. Meanwhile, there appears a vortex adjacent to the sidewall on the leeward side.

Additionally in Fig. 13, pressure distributions are plotted on the external and internal walls, denoted by black and orange colors respectively; it is noted that the high magnitude portion of the internal surface pressure turns out to belong to the upper surface. By and large both inlets behave similarly; some differences can still be discerned. At the entrance a noticeable difference in pressure is detected on the nacelle external surface, where the optimized inlet gives a relatively leveled distribution albeit somewhat bumpy. At $X / D_{2}=0.5$, the lower surface of the optimized inlet also has more nonuniformity, explaining the earlier appearance of the low speed pocket mentioned above.

Next we study the oil flow representations on floor surfaces in Fig. 14 for both baseline and optimized inlets. Both are again similar, except inside the inlet entrance the optimized inlet exhibits spatial variations caused by modulating the wall shape. Both inlets reveal that the flow is significantly displaced outward from the nacelle in the windward side, resulting from the front flow separation discussed above regarding Fig. 13, The leeward side has got a large flow separation $\left(X / D_{2} \approx 2\right.$, see Fig. 13), as a result the flow is greatly displaced away from the nacelle. There is a saddle-like singularity, located near the lip region respectively on the windward and leeward sides. The oil pattern on the bottom flow of the inlet is constricted considerably immediately after entering the inlet, showing a lateral push on the streamlines away from the wall. By comparing with the case of no crosswind, as given in Fig. 5, we notice that the constriction at the low speed condition does not seem to be as severe inside the inlet as at high speed condition on cruise. Thus the total pressure recovery is higher at low speed.

Finally, we show three views of oil flow patterns on the outer surface of the nacelle in Fig. 15, respectively: (1) the top view 

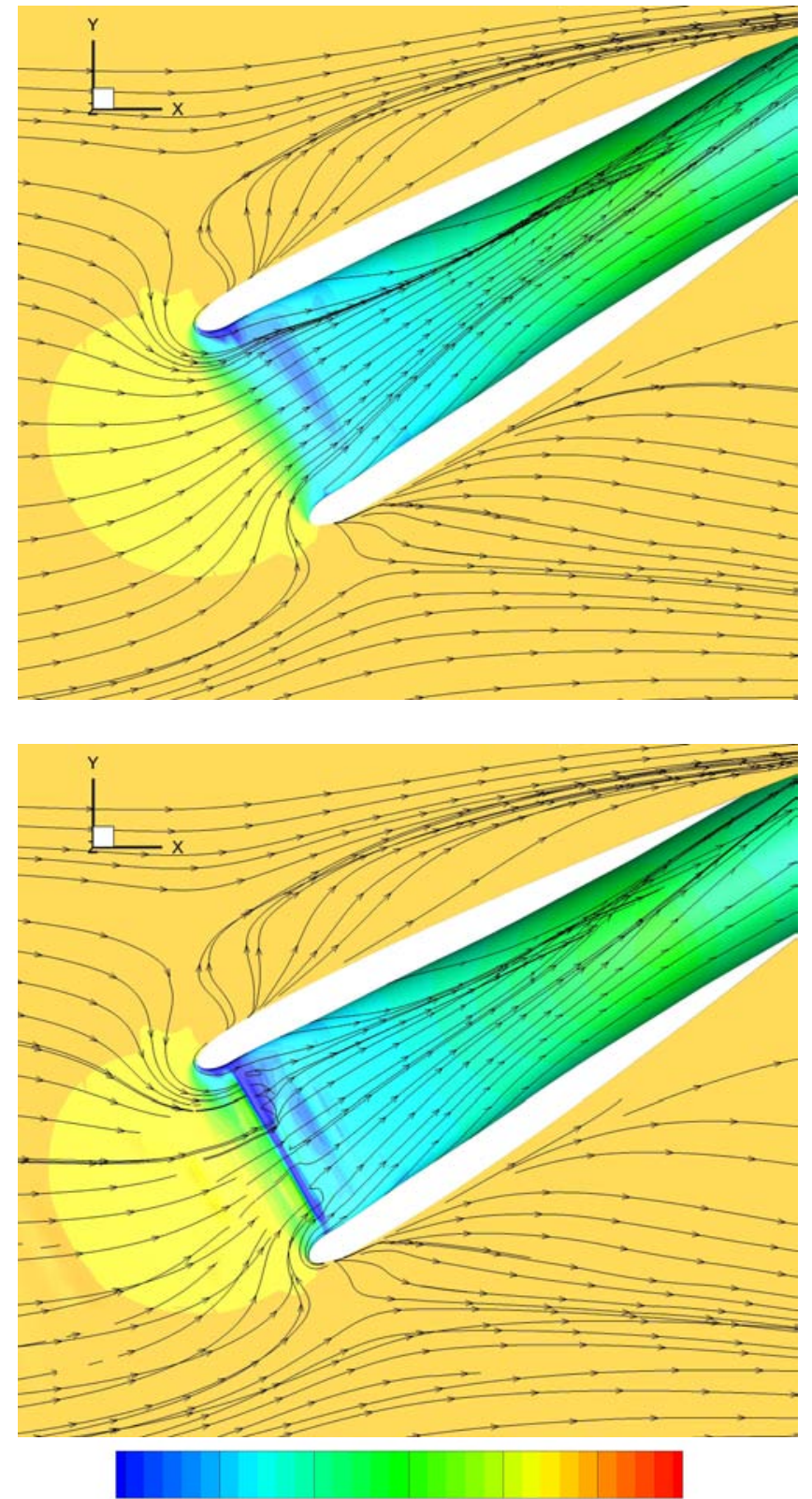

p: $0.400 \quad 0.462 \quad 0.524 \quad 0.5860 .648 \quad 0.710$

FIGURE 14: OIL FLOW PATTERNS ON THE BOTTOM WALL OF INLET AND BOTTOM PLATE SUPERIMPOSED WITH PRESSURE CONTOURS. TOP: BASELINE INLET; BOTTOM: OPTIMIZED INLET.

in the middle plot, (2) the sideview from the windward side in the top plot, and (3) the sideview from the leeward side in the bottom plot. A top view of the oil flow pattern on the plate is also included. The flow on the nacelle top surface behaves noticeably different from that on the inlet bottom wall (seen above
(3)

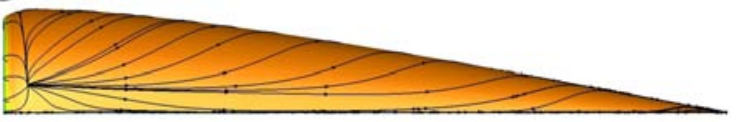

(1)

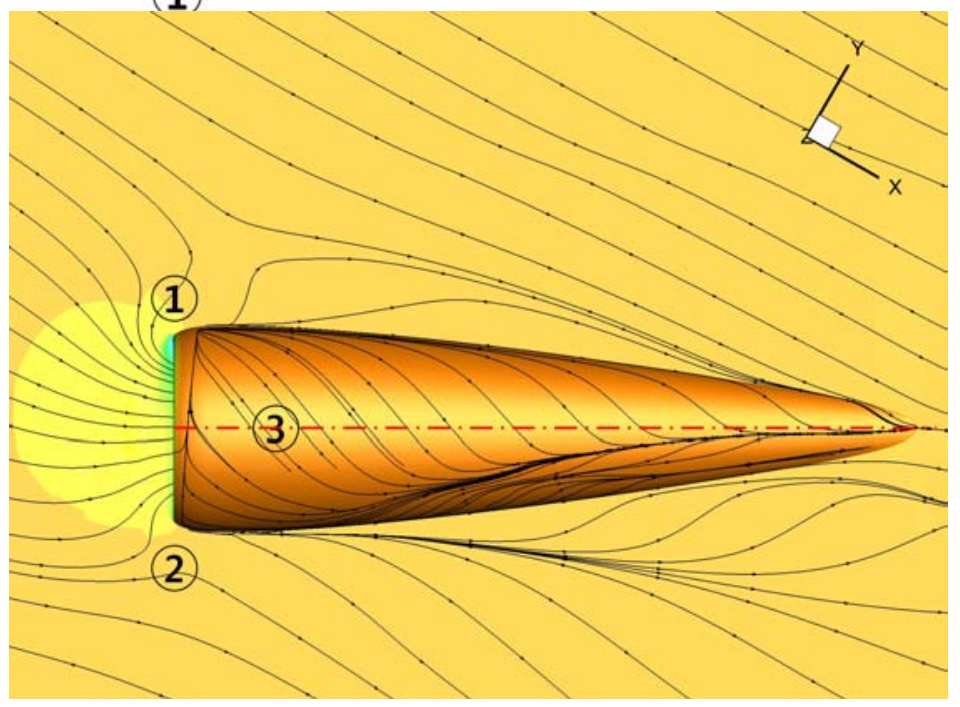

(3)

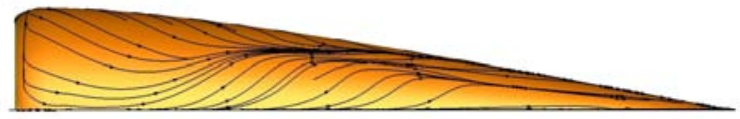

(2)

\section{p: $\quad \begin{array}{llllll}0.400 & 0.462 & 0.524 & 0.586 & 0.648 & 0.710\end{array}$}

FIGURE 15: OIL FLOW PATTERNS ON NACELLE EXTERNAL SURFACES SUPERIMPOSED WITH PRESSURE CONTOURS. TOP: WINDWARD SIDE VIEW; MIDDLE: TOP VIEW; AND BOTTOM: LEEWARD SIDE VIEW.

in Fig. 147; the flow from windward side towards leeward side is seen, but the flow separation on the leeward side strongly pushes back, thereby showing converging of particle traces. Also, the two flow separation, each on the windward and leeward sides, shows the effects on the bending of particle traces. Similar behavior is observed in the optimized inlet in these views, hence they are omitted. 


\section{CONCLUDING REMARKS}

Motivated by NASA's interest in pursuing next generations of civil transport to environment and performance metrics, we have focused on the so-called N2B configuration which is a hybrid (blended) wingbody with a top-mounted propulsion system into which a boundary layer of considerable thickness relative to the inlet height will be swallowed and diffused in an offset duct, resulting in a flow much more severely distorted than in the current fleet. This distortion results from a combination of factors, such as vorticity embedded in the incoming boundary layer, lip separation, adverse pressure gradient, secondary flow, etc, all manifested into a enormously complicated flow phenomenon. Hence, it can be only analyzed via a high-fidelity setting to shed insight into the underlying flow physics. To keep flow distortion at the AIP within a tolerable level, some forms of control over the flow must be instituted, either by conventional (such as vortex generators, active plasma/synthetic jet, etc.) or other innovative ideas. Our previous studies show that it is incredibly effective to reduce flow distortion and increase total pressure recovery at the engine face by conditioning/guiding the flow through modification of wall shape, instead of locally controlling through added penalizing devices.

The objective of the present study is to assess the performance of the boundary-layer-ingesting inlet when subject to cross wind at low speed flight, e.g., during takeoff or landing. We focus on investigating the flow characteristics unique to this condition. Moreover, we are also interested in quantifying the characteristics in the inlet optimized for the cruise condition and comparing the differences in performance between the original baseline and the optimized inlets. In this investigation, we have shown that the flow in this off-design condition is very complex, involving additional flow separations external to nacelle, which will thus affect the aerodynamic performance of the aircraft to which the inlet is installed. As the flow enters the inlet with a crosswind component, its effect seems to last within a short distance and quickly subsides by the constraining boundary of the inlet. At the low speed condition, the inlet distortion is low even with the crosswind effect, especially in the baseline inlet. The present study concludes that insofar as the inlet distortion is concerned, the crosswind effect, even up to 40 knots, is probably not of concern to operation of an engine fan. The pressure recovery in fact remains high, because of low losses owing to low speed flow. However, the distortion in the optimized inlet is several times higher than that of baseline inlet, although the pressure recovery is only slightly lower. This suggests that a revisit to our previous optimization may be warranted; it is a follow-on investigation that will be reported in a subsequent part of the series on the BLI inlet. In fact, this aligns with our view that for an effective mitigation of distortion in a BLI inlet system, a design not only considering the inlet passage itself, but also combining the airframe geometry surrounding the nacelle, is necessary.

\section{ACKNOWLEDGMENT}

This paper presents part of the multidisciplinary design analysis and optimization (MDAO) effort contributing to the research towards next generation transport sponsored by the subsonic fixed wing (SFW) project, under the fundamental aeronautics program (FAP) in NASA's Aeronautics Mission Directorate. The authors are grateful for the support of SFW management team.

\section{REFERENCES}

[1] Lee, B. J., and Liou, M.-S., 2010. "Optimizing a boundarylayer-ingesting offset inlet by discrete adjoint approach". AIAA Journal, 48(9), pp. 2008-2016.

[2] Liou, M.-S., and Lee, B. J., 2012. "Minimizing inlet distortion for hybrid wing body aircraft". J. of Turbomachinery, 134.

[3] NASA. http://www.hq.nasa.gov/office/aero/.

[4] Smith, A., and Robert, H., 1947. "The jet airplane utilizing boundary air for propulsion". J. Aeronaut. Sci., 14, pp. 97109.

[5] Lynch, F. T., 1960. A theoretical investigation of the effect of ingesting airframe boundary layer air on turbofan engine fuel consumption. Douglas aircraft company, texh. report sm-23981.

[6] Douglass, W. M., 1970. Propulsion efficiency with boundary layer ingestion. Tech. rep., MDC J0860, McDonnell Douglas Corp., Long Beach, CA.

[7] Liebeck, B. H., 2002. Design of the blend-wing-body subsonic transport. AIAA Paper 2002-002, Jan.

[8] Cambridge-MIT Institute. http://silentaircraft.org/.

[9] European Commission. http://ec.europa.eu/research/researchfor-europe/transport-nacre_en.html.

[10] Nickol, C. L., and McCullers, L. A., 2009. Hybrid wing body configuration studies. AIAA Paper 2009-931, Jan.

[11] Daggett, D. L., Kawai, R., and Friedman, D., 2003. Blended wing body systems studies: boundary layer ingestion inlets with active flow control. NASA CR 2003212670.

[12] Plas, A. P., et al., 2007. Performance of a boundary layer ingestng (BLI) propulsion sustem. AIAA Paper 2007-0450, Jan.

[13] Hall, C. A., Schwartz, E., and Hileman, J. I., 2009. "Assessment of techhnologies for the silent aircraft initiative". J. Propulsion and Power, 25(6), pp. 1153-1162.

[14] Godard, J.-L., 2010. Semi-buried engine installation: the nacre project experience. 27th international congress of aeronautical sciences, icas2010.

[15] Carter, M. L., et al., 2006. "Designing and testing of a blended wing body with boundary-layer ingestion nacelles". AIAA Journal, 43, pp. 1479-1489.

[16] Anabtawi, A. J., et al., 1999. An experimental investigation of boundary layer ingestion in diffusing s-duct with 
and without passive flow control. AIAA Paper 1999-0739, Jan.

[17] Berrier, B. L., Carter, M. L., and Allan, B. G., 2005. High reynolds number investigation of a flush-mounted, sduct inlet with large amounts of boundary layer ingestion. NASA TP 2005-213766.

[18] Anderson, B., and Gibb, J., 1998. "Vortes-generator installation studies on steady-state and dynamic distortion". J. Aircraft, 35, pp. 513-520.

[19] Allan, B. G., and Owens, L. R., 2006. Numerical modeling of flow control in a boundary-layer-ingesting offset diffuser at transonic mach number. AIAA Paper 2006-0845, Jan.

[20] Gorton, S. A., et al., 2004. Active flow control on a boundary-layer-ingesting inlet. AIAA Paper 2004-1203, Jan.

[21] Lee, B. J., Kumano, T., and Liou, M.-S., 2010. Design exploration for vortex generators for boundary-layeringesting inlet. AIAA Paper 2010-9399.

[22] Hall, C. A., and Hynes, T. P., 2005. "Nacelle interaction with natural wind before takeoff". J. Propulsion and Power, 21(5), pp. 784-791.

[23] Hall, C. A., and Hynes, T. P., 2006. "Measurements of intake separation hysteresis in a model fan and nacelle rig". J. Propulsion and Power, 22(4), pp. 872-879.

[24] Collin, Y., et al., 2007. Numerical simulation and anslysis of crosswind inlet flows at low mach numbers. ISAIF80058, 8th International Symposium on Experimental and Computational Aerodynamics of Internal Flows, Lyon, France, July.

[25] Menter, F., 1994. "Two-equation eddy-viscosity turbulence models for engineering applications". AIAA Journal, 32, pp. 1598-1605.

[26] Lee, B. J., 2007. “Aerodynamic shape optimization for internal and external flows via discrete adjoint approach and overset mesh system". PhD Thesis, Seoul National University, Seoul, S. Korea.

[27] Liou, M.-S., 2006. "A sequel to AUSM, part II: AUSM+-up for all speeds". J. Comput. Phys., 214, pp. 137-170.

[28] Yoon, S. K., and Jameson, A., 1988. "Lower-upper symmetric-gauss-seidel method for the euler and navierstokes equations". AIAA Journal, 26, pp. 1025-1026.

[29] Benek, J. A., et al., 1986. A grid-embedding technique. Aedc-tr-85-64.

[30] Berrier, B. L., and Allan, B. G., 2004. Experimental and computational evaluation of flush-mounted, s-duct inlets. AIAA Paper 2004-1203.

[31] SAE, 2010. Gas turbine engine inlet flow distortion. Society of Automotive Engineers ARP-1420, Jan. 